\title{
Electronic $g$ factor and magnetotransport in InSb quantum wells
}

\author{
Zijin Lei $\odot,{ }^{1, *}$ Christian A. Lehner $\odot,{ }^{1}$ Km Rubi $\odot,{ }^{2}$ Erik Cheah, ${ }^{1}$ Matija Karalic $\odot,{ }^{1}$ Christopher Mittag, ${ }^{1}$ Luca Alt $\odot,{ }^{1}$ \\ Jan Scharnetzky, ${ }^{1}$ Peter Märki ${ }^{\circledR},{ }^{1}$ Uli Zeitler, ${ }^{2}$ Werner Wegscheider, ${ }^{1}$ Thomas Ihn ${ }^{\circledR},{ }^{1}$ and Klaus Ensslin ${ }^{1}{ }^{1}$ \\ ${ }^{1}$ Solid State Physics Laboratory, ETH Zurich, CH-8093 Zurich, Switzerland \\ ${ }^{2}$ High Field Magnet Laboratory (HFML-EMFL), Radboud University, 6525 ED Nijmegen, The Netherlands
}

(Received 12 February 2020; revised 26 May 2020; accepted 15 July 2020; published 7 August 2020)

\begin{abstract}
High mobility InSb quantum wells with tunable carrier densities are investigated by transport experiments in magnetic fields tilted with respect to the sample normal. We employ the coincidence method and the temperature dependence of the Shubnikov-de Haas oscillations and find a value for the effective $g$ factor of $\left|g^{*}\right|=35 \pm 4$ and a value for the effective mass of $m^{*} \approx 0.017 m_{e}$, where $m_{e}$ is the free electron mass. Our measurements are performed in a magnetic field and a density range where the enhancement mechanism of the effective $g$ factor can be neglected. Accordingly, the obtained effective $g$ factor and the effective mass can be explained in a single-particle picture. Additionally, we explore the magnetotransport up to magnetic fields of $35 \mathrm{~T}$ and do not find features related to the fractional quantum Hall effect.
\end{abstract}

DOI: 10.1103/PhysRevResearch.2.033213

The narrow-gap III-V binary compound InSb is well known for its combination of a light effective mass, high electron mobility, strong spin-orbit interactions, and a giant effective $g$ factor in the conduction band [1-6]. These unique properties are interesting in view of potential applications such as high-frequency electronics [7], optoelectronics [8], and spintronics [9]. The large $g$ factor with a bulk value of $\left|g^{*}\right| \sim 51$ could be advantageous for hosting a topologically nontrivial phase through proximity-induced superconductivity $[10,11]$. This has led to research efforts in nanomaterials and sophisticated nanoconstrictions. In two-dimensional systems, so-called coincidence measurements have been used in $\mathrm{InSb}$ quantum wells (QWs). Here the magnetic field is tilted with respect to the sample surface normal to tune the relative strength of Zeeman and Landau-level splitting $[12,13]$. The effective $g$ factor has been measured in nanoconstrictions too, such as in nanowire-based quantum dots $[14,15]$, and in QW-based quantum point contacts $[16,17]$.

Nevertheless, most of the previous experimental results focused on the situations of special quantum regimes or with relatively low Landau-level filling factors where exchange effects dominate. These lead to an effective $g$ factor far off from the predictions of $\boldsymbol{k} \cdot \boldsymbol{p}$ theory. For example, in InSb nanowire-based quantum dots, the effective $g$ factor is more than $40 \%$ larger than that in the bulk material because of the level-to-level fluctuations arising from spin-orbit interaction $[14,18]$. Caused by the confinement of the nanoconstrictions, the effective $g$ factor measured in chemically etched defined quantum point contacts (QPCs) shows strong reductions and

\footnotetext{
*Corresponding author: zilei@phys.ethz.ch

Published by the American Physical Society under the terms of the Creative Commons Attribution 4.0 International license. Further distribution of this work must maintain attribution to the author(s) and the published article's title, journal citation, and DOI.
}

large anisotropies $[16,17]$. Limited by the low carrier mobilities, the coincidence measurement in a tilted magnetic field can only be accomplished at a relatively low filling factor. The large spin polarization leads to a substantial enhancement of the $g$ factor as a result of exchange interactions $[12,19]$. For instance, in Ref. [12], the effective $g$ factor is enhanced linearly as a function of spin polarization over a range of filling factors $v=2-7$ Different from the results listed above, the bare $g$ factor deserves to be investigated more for the research related to topological superconductivity, because the band gap should be opened by the Zeeman splitting while applying a relatively small parallel magnetic field [20]. Efforts have been made for this purpose. For example, Ref. [13] arrived at extracting a bare $g$ factor of $\sim 39$ in a 20-nm-wide InSb/InAlSb QW by extrapolating data measured in the finite spin polarization regime to the zero polarization limit. Furthermore, recent measurement results from purely gate-defined quantum dots fabricated on InSb QWs present the estimation of the effective $g$ factor of a value between 26 and 35 [21]. This is close to the bare effective $g$ factor resulting from the $\boldsymbol{k} \cdot \boldsymbol{p}$ theory. However, the accuracy is still limited by the quality of the devices. In the experiment presented in this paper, we show a precise measurement of the bare $g$ factor by performing a coincidence measurement for InSb two-dimensional electron gas (2DEG) confined between InAlSb barriers. The high electron mobilities (up to $3 \times 10^{5} \mathrm{~cm}^{2} / \mathrm{V} \mathrm{s}$ ) enable us to investigate the dependence of the Shubnikov-de Haas $(\mathrm{SdH})$ oscillations on the tilt angle of the magnetic field relative to the plane of the 2DEG in a relatively small total magnetic field and for large filling factors. With the combination of a precise measurement of the effective mass, the effective $g$ factor of the InSb 2DEG turns out to be $\left|g^{*}\right|=35 \pm 4$, which can be quantitatively explained with a single-particle calculation based on $\boldsymbol{k} \cdot \boldsymbol{p}$ theory. Finally, we present electron transport in magnetic fields as high as $35 \mathrm{~T}$. We find no signatures related to the fractional quantum Hall effect which asks for further improvement of the sample quality in the future. 
(a)

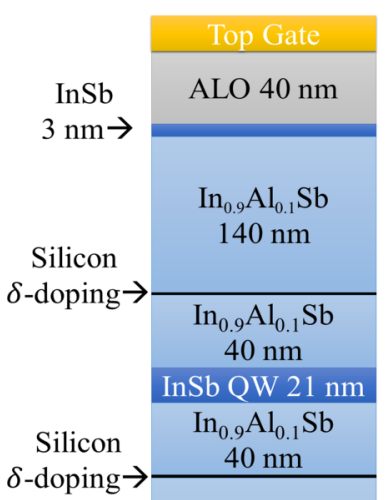

(c)

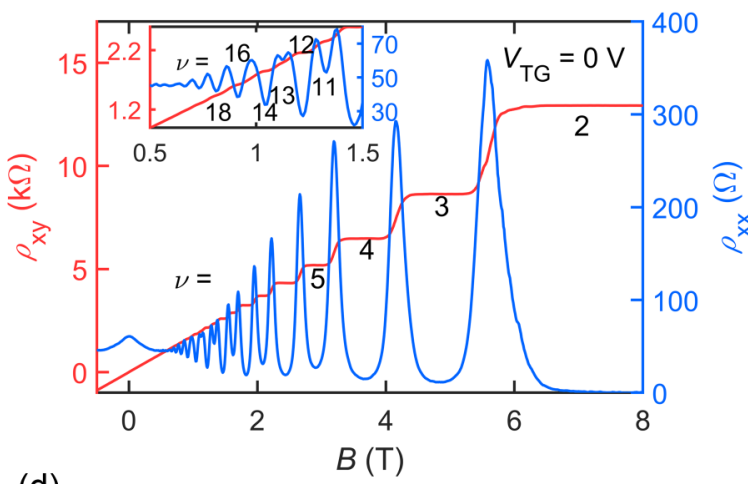

(d)

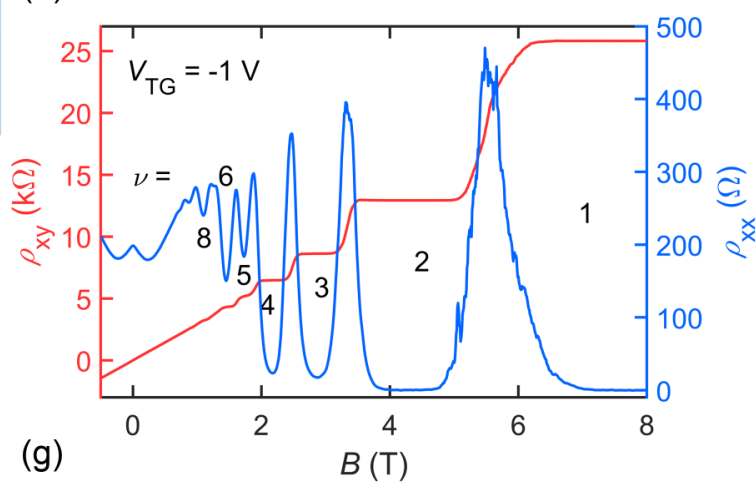

(e)

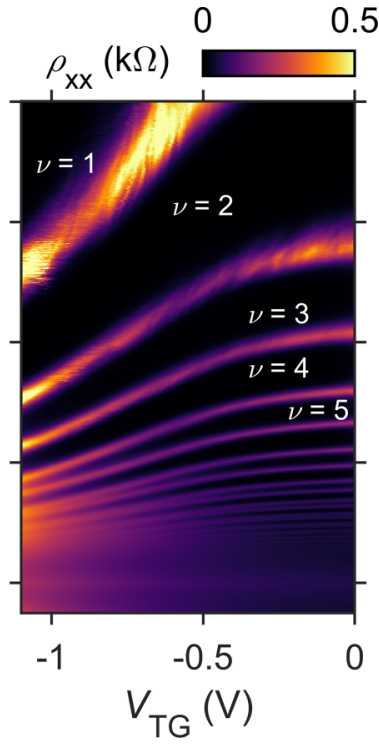

(b)

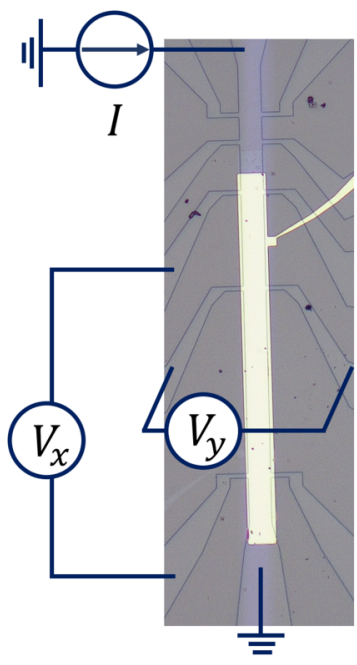

$\mathrm{In}_{0.9} \mathrm{Al}_{0.1} \mathrm{Sb}$

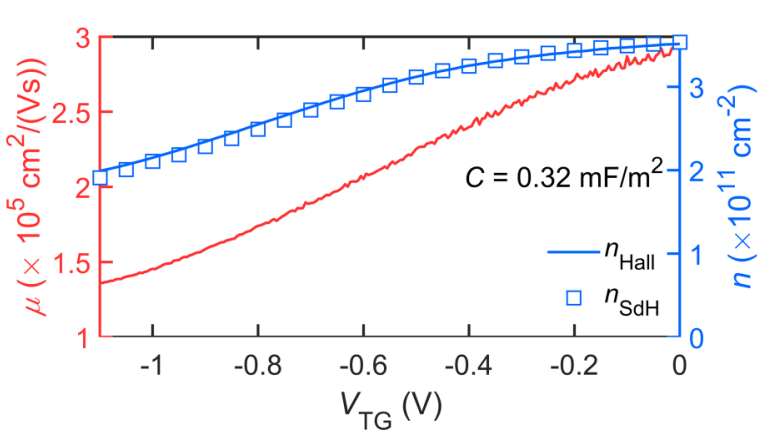

(f)

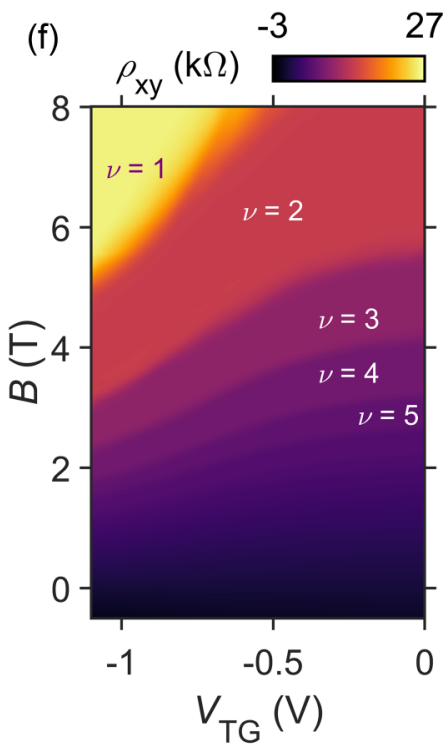

FIG. 1. (a) Layer structure of the QW. (b) An optical image of the Hall bar sample. The measurement setup is added schematically. (c), (d) The $B$ dependence of $\rho_{x x}$ (blue) and $\rho_{x y}$ (red) for $V_{\mathrm{TG}}=0 \mathrm{~V}$ and $V_{\mathrm{TG}}=-1 \mathrm{~V}$ are presented, respectively. The filling factors are labeled on the plateaus of the Hall traces or the minima of the $\mathrm{SdH}$ oscillations. Inset of (c): the zoom-in of (c) in the small magnetic field range. The axes are the same with (c) and (d). (e), (f) The detailed transport characterization at $1.3 \mathrm{~K}$ with $\rho_{x x}$ (e) and $\rho_{x y}$ (f) as functions of $V_{\mathrm{TG}}$ and $B$. The filling factors are labeled in white color on the Landau Fan diagrams. (g) The blue line and squares show the carrier density of the Hall bar obtained from the Hall effect $n_{\mathrm{Hall}}$ and from the $1 / B$ periodicity of the $\mathrm{SdH}$ oscillations $n_{\mathrm{SdH}}$ plotted against $V_{\mathrm{TG}}$. The red line depicts the dependence of the electron mobility $\mu$ on $V_{\mathrm{TG}}$.

The InSb QW sample we investigate here is grown on a (100) GaAs substrate by molecular beam epitaxy (MBE). A schematic layer sequence is shown in Fig. 1(a). A specialized interfacial misfit transition to a GaSb buffer and an interlayer InAlSb buffer is employed to overcome the lattice mismatch between GaAs and InSb. The total thickness of the buffer system amounts to roughly $3 \mu \mathrm{m}$. Then, the 21-nm-thin InSb quantum well is surrounded by $\mathrm{In}_{0.9} \mathrm{Al}_{0.1} \mathrm{Sb}$ confinement barriers, while the $n$-type carriers are introduced to the active region by two $\mathrm{Si} \delta$-doping layers incorporated $40 \mathrm{~nm}$ below and above the QW in the barrier, respectively. On the top of the $\mathrm{QW}$, an $\mathrm{In}_{0.9} \mathrm{Al}_{0.1} \mathrm{Sb}$ layer with a thickness of 180 $\mathrm{nm}$ is grown. Finally, a 3-nm-thick InSb capping layer is employed on the top of the sample to prevent possible oxi- dization. More details about the MBE growth can be seen in Ref. [22].

The microfabrication process is similar to our previous work [23]. A standard Hall bar sample is defined using wet chemical etching with an etching depth of more than $270 \mathrm{~nm}$, which is deeper than the $\mathrm{Si} \delta$-doping layer on the substrate side. Layers of $\mathrm{Ge} / \mathrm{Ni} / \mathrm{Au}$ evaporated after an Ar milling provide the Ohmic contacts. The sample is coated with a 40-nm-thick aluminum oxide (ALO) dielectric layer using atomic layer deposition (ALD) at a temperature of $150{ }^{\circ} \mathrm{C}$. A high-temperature annealing step is unnecessary because the metal diffuses into the 2DEG during the heating during the ALD process. Finally, a Ti/Au top gate covering the Hall bar is deposited with electron-beam evaporation. 


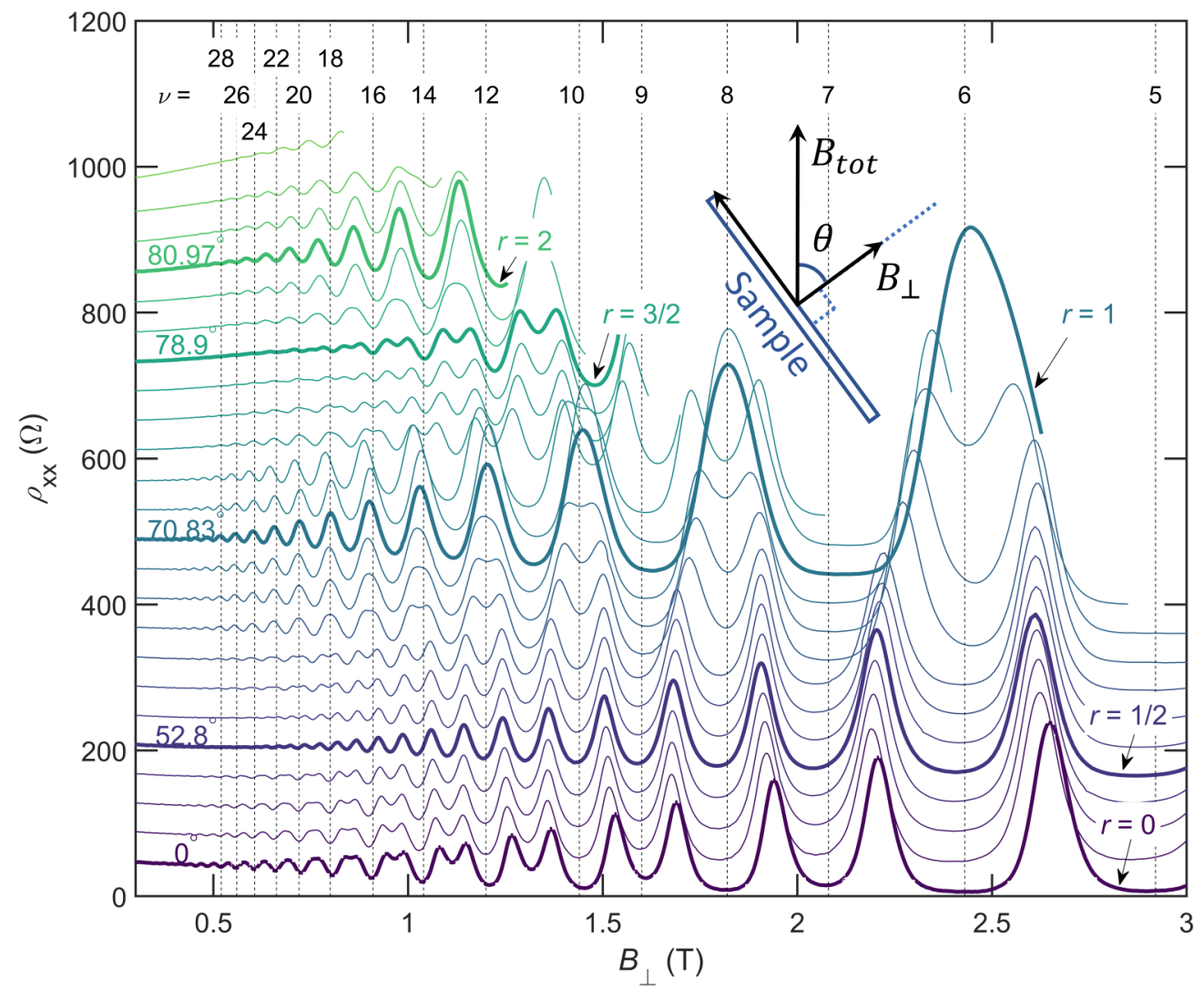

FIG. 2. The SdH oscillations measured with different tilt angles. The traces have a constant offset of $40 \Omega$. The traces with $r=0,1 / 2,1$, and 2 are high lighted with thick lines, together with the corresponding angles. The integer filling factors are labeled with dashed lines. Inset: The definition of the tilt angle $\theta$.

Figure 1(b) presents an optical image of the sample together with the schematic diagram of the magnetotransport measurement configuration. The part of the Hall bar we measured has a size of $310 \times 25 \mu \mathrm{m}^{2}$. The transport measurement is accomplished by using standard low frequency $(77 \mathrm{~Hz})$ lock-in techniques in a cryostat with a base temperature of $1.3 \mathrm{~K}$ and a rotatable magnetic field up to $8 \mathrm{~T}$. Figures 1(c) and 1(d) show the longitudinal and the transverse resistivities $\rho_{x x}$ and $\rho_{x y}$ where the top gate voltages $V_{\mathrm{TG}}$ are 0 and $-1 \mathrm{~V}$, respectively. Pronounced $\mathrm{SdH}$ oscillations in $\rho_{x x}$ and quantum Hall plateaus in $\rho_{x y}$ at $h / v \mathrm{e}^{2}$ are visible, where $v$ is the filling factor. The inset in Fig. 1(c) shows that the Zeeman splitting starts from magnetic fields around 1.1 T. This measurement indicates that a single-channel 2DEG system with a tunable density and a high mobility is embedded in the QW. Figures 1(e) and 1(f) show the Landau fan diagrams of $\rho_{x x}$ and $\rho_{x y}$, i.e., the dependence of $\rho_{x x}$ and $\rho_{x y}$ on perpendicular magnetic field $B$ and $V_{\mathrm{TG}}$. The slight bending of the Landau fan diagram at high $V_{\mathrm{TG}}$ may be caused by the charging of the capping layer or the barrier layer above the QW. The carrier density $n$ is calculated through both the linear fitting of the Hall effect in a small magnetic field window (from -0.3 to $0.5 \mathrm{~T}$ ) and the $1 / B$ periodicity of the $\mathrm{SdH}$ oscillations. As shown in Fig. $1(\mathrm{~g})$, the carrier density can be tuned from $2 \times$ $10^{11} \mathrm{~cm}^{-2}$ to $3.5 \times 10^{11} \mathrm{~cm}^{-2}$ while increasing the mobility from $1.3 \times 10^{5} \mathrm{~cm}^{2} /(\mathrm{V} \mathrm{s})$ to $3 \times 10^{5} \mathrm{~cm}^{2} /(\mathrm{V} \mathrm{s})$. The gate capacitance is estimated to be $C=0.32 \mathrm{mF} / \mathrm{m}^{2}$ at the linear part of the $n-V_{\mathrm{TG}}$ line, which is about $40 \%$ less than the value calculated from the plane-parallel capacitor model, which is similar to the results of our previous work [23] and Ref. [21] by another group.

In the following, we present our results of the coincidence measurement when $n=3.45 \times 10^{11} \mathrm{~cm}^{-2}$ at $V_{\mathrm{TG}}=$ $-0.1 \mathrm{~V}$. Similar strategies were used for QWs with large effective $g$ factors before, such as $\mathrm{Si} / \mathrm{SiGe}$ [24] and InAs [25]. The Landau-level spacing is proportional to the perpendicular magnetic field $B_{\perp}$, but the Zeeman energy is proportional to the total magnetic field $B_{\text {tot }}$. We introduce a parameter $r$ as the ratio between the Zeeman energy and the Landau-level spacing, i.e., $r=\left|g^{*}\right| \mu_{\mathrm{B}} B_{\mathrm{tot}} / \hbar \omega_{c}$. Here $\omega_{c}=e B_{\perp} / m^{*}, m^{*}$ is the effective electron mass, $\mu_{\mathrm{B}}$ is the Bohr magneton, and $B_{\text {tot }}$ is the total magnetic field applied. The inset of Fig. 2 shows the definition of the tilt angle $\theta$, where $\theta=0$ corresponds to a magnetic field perpendicular to the sample surface and $\theta=90^{\circ}$ means an in-plane magnetic field. With these, we can achieve $B_{\perp}=\cos (\theta) B_{\text {tot }}$ and $r \cos (\theta)=\left|g^{*}\right| m^{*} / 2 m_{e}$, where $m_{e}$ is the free-electron mass. By increasing the angle $\theta$ to $90^{\circ}, r$ can be increased continuously. The range of values of $r$ and $\theta$ is experimentally limited by the finite perpendicular field component $B_{\perp}$ required to detect $\mathrm{SdH}$ oscillations. It shows the idea of the coincidence method that $\left|g^{*}\right| m^{*}$ can be extracted if we can find situations where both $r$ and $\cos (\theta)$ are precisely measured. The inset in Fig. 3(b) describes the various coincidence situations which are characterized by the parameter $r$ schematically. At $r=1 / 2$ and 3/2, the Landau levels with both even and odd filling factors are separated 


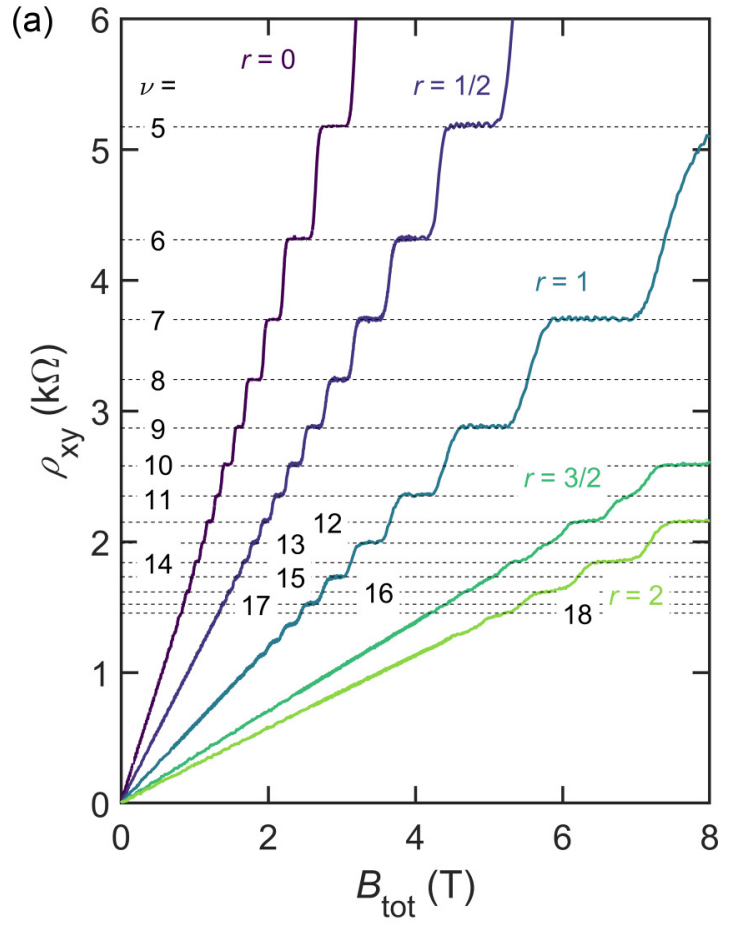

(b)

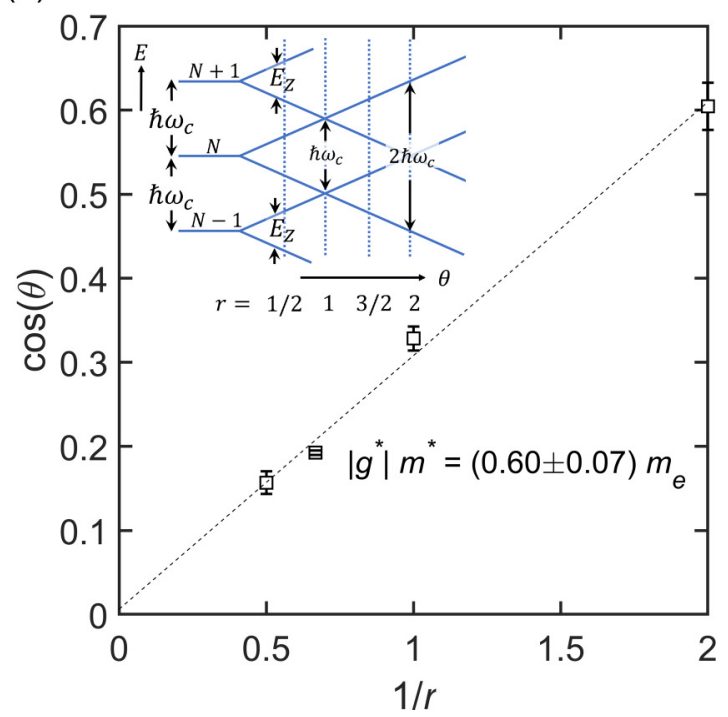

FIG. 3. (a) The quantum Hall effect traces with $r=0,1 / 2$, 1, and 2. The Hall plateaus are labeled with the dashed lines. (b) Coincidence plot gathered from the angles in Fig. 2. The straight line has a slope indicating $\left|g^{*}\right| m^{*}=(0.60 \pm 0.07) m_{e}$. Inset: the various coincidence situations which are characterized by the parameter $r$, the ratio of the Zeeman and the cyclotron energies. The energy gaps between even and odd Landau states get opened and closed periodically.

by the same energy. Consequently, the minima of $\mathrm{SdH}$ traces will occur at both even and odd integer filling factors. On the other hand, for $r=1$ or 2, because two Landau states with opposite spin directions will have the same energy, the energy gap between them gets closed. Thus, the longitudinal resistivity minima only occur at odd or even integer filling factors. In our experiment, we determine the situations with the most obvious $\rho_{x x}$ minima at both even and odd filling factors to be $r=1 / 2$ and $3 / 2$ and the situation with the most pronounced $\rho_{x x}$ minima at only even or odd filling factors to be $r=1$ or 2 . Figure 2 depicts the measurement results showing the dependence of $\rho_{x x}$ on $B_{\perp}$ for a continuous change in $\theta$ from $0^{\circ}$ to $84^{\circ}$. The value of $\theta$ is calibrated with the slope of the Hall effect in a low magnetic field with a high accuracy of better than $0.1^{\circ}$. The thick lines in Fig. 2 show magnetoresistance traces at specific $\theta$ values where the $\mathrm{SdH}$ minima occur only at even integer filling factors $(r=2)$, at even and odd filling factors $(r=1 / 2,3 / 2)$, or only at odd-integer filling factors $(r=1)$. The trace with a purely perpendicular magnetic field $(\theta=0, r=0)$ is labeled with a thick line too. Similar to the $\mathrm{SdH}$ traces, the disappearance and the reappearance of the Hall plateaus at even and odd filling factors can be investigated as a function of increasing $\theta$. As shown in Fig. 3(a), both even and odd filling factor plateaus can be seen when $r=1 / 2$ and $3 / 2$, but only odd or even filling factor plateaus are observed when $r=1$ or 2 .

One can determine the product of $g$ factor and effective mass by taking these results into consideration. Figure 3(b) shows the relation between $1 / r$ and the corresponding $\cos (\theta)$. The error bar is determined by the space of $\theta$ values between the traces with the angles of coincidence $(r=1 / 2,1,3 / 2$, and 2) and the neighboring traces which do not show the coincidence. The accuracy of our measurement is mainly limited by the broadening of the Landau levels, especially when $r=1 / 2$ and $r=3 / 2$, where the SdH oscillations are not very pronounced in a low magnetic field regime. This linear relation indicates that $\left|g^{*}\right| m^{*}$ and therefore $\left|g^{*}\right|$ are constant within our measurement range, i.e., exchange enhancement effects are not relevant. The slope of the plot shows that the product of $\left|g^{*}\right| m^{*}=(0.60 \pm 0.07) m_{e}$. Thus, only the effective mass is left to measure in order to extract the value of $\left|g^{*}\right|$.

In the following, we present the measurement of the effective mass in the same sample through the temperature dependence of the SdH oscillations in small magnetic fields. With the increase of the measurement temperature, the amplitude of the SdH oscillations will decrease until the oscillations vanish completely. The lower the effective mass is, the higher the temperature at which the $\mathrm{SdH}$ oscillations can still be observed. This temperature dependence of the $\mathrm{SdH}$ oscillation is well described by a formula in a small magnetic field regime [26]. Figure 4(a) shows the $\mathrm{SdH}$ oscillations for a carrier density $n=3.44 \times 10^{11} \mathrm{~cm}^{-2}$ measured at temperatures from 1.53 to $15 \mathrm{~K}$. The oscillating part of the resistivity $\Delta \rho_{x x}$ is obtained by subtracting the background of the magnetoresistance $\bar{\rho}_{x x}$. Here we select the local maxima and minima in the traces of $\Delta \rho_{x x}$ vs $B$ as the data points to depict the envelope of the $\mathrm{SdH}$ oscillations. Figure 4(b) presents the fit of the Dingle factor to $\ln \left(\Delta \rho_{x x} T_{0} / \bar{\rho}_{x x} T\right)$, where $T_{0}$ is the lowest temperature at which we measured the $\mathrm{SdH}$ oscillations [26]. The obtained effective mass is $m^{*} \approx 0.017 m_{e}$ with an error smaller than $0.001 m_{e}$ within the magnetic field range of the measurement [see the inset of Fig. 4(c)]. Figure 4(c) shows the fitting to obtain the quantum lifetime using the effective mass discussed above. We extract a quantum lifetime $\tau_{q}=0.10 \pm 0.01 \mathrm{ps}$ from the slope obtained from the linear fitting of the plotting of $1 / B$ vs $\ln \left(\frac{\Delta \rho_{x x}}{\bar{\rho}_{x x}} f(B, T)\right)$, where 
(a)

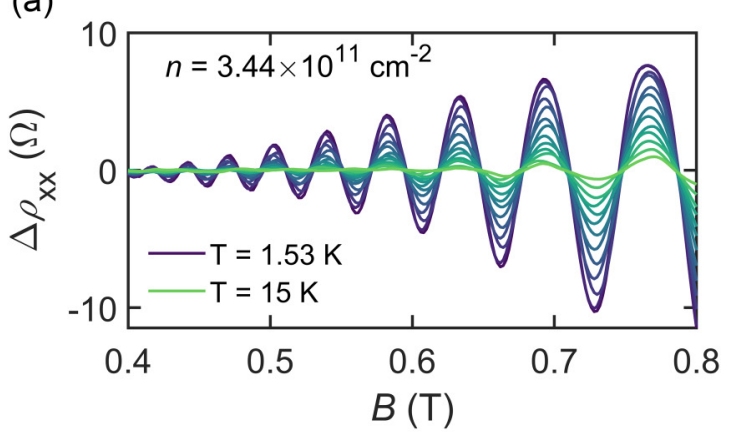

(b)

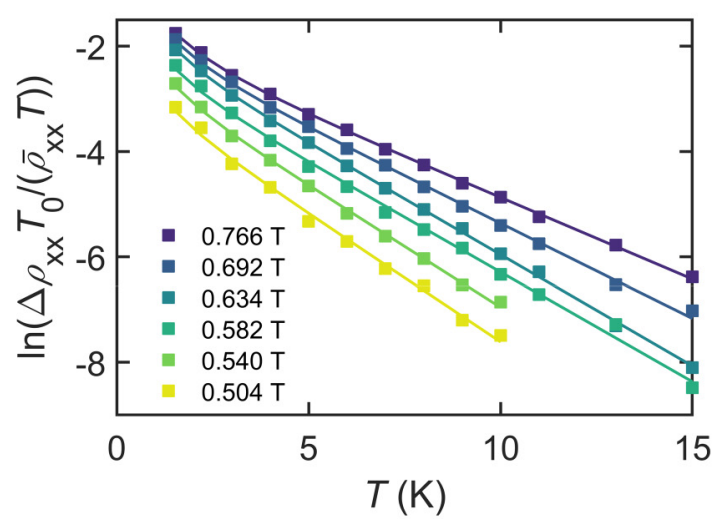

(c)

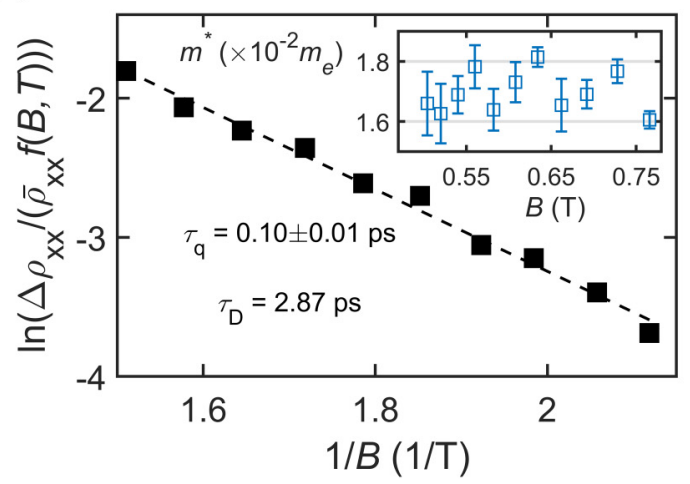

FIG. 4. Effective mass measurement. (a) Temperaturedependence of $\mathrm{SdH}$ oscillations with $n=3.44 \times 10^{11} \mathrm{~cm}^{-2}$. (b) Dingle factor fitting with different $B$. The squares are data and the lines are fitted curves. (c) The fitting of the quantum lifetime. Inset: The effective mass obtained from the Dingle factor fitting vs $B$.

$f(B, T)=\left(2 \pi^{2} k_{B} T / \hbar \omega_{c}\right) / \sinh \left(2 \pi^{2} k_{B} T / \hbar \omega_{c}\right)$. Considering the Drude scattering time $\tau_{D}=2.87 \mathrm{ps}$ calculated from the Drude model at this carrier density, the Dingle ratio can be calculated as $\tau_{D} / \tau_{q} \approx 29$. This value, which is considerably larger than 1 , indicates that our 2DEG is in the regime where the dominant scattering mechanism originates from long-range potential fluctuations [26-28]. On the other hand, we observe that $\mu \propto n$ from magnetotransport measurements. The linear relationship between $\mu$ and $n$ was also observed in a high-quality asymmetric InSb QW in Ref. [4]. This implies that background impurity scattering is dominant [29] which leads to a short-range scattering potential. These two observations, the large Dingle ratio on the one hand and the linear behavior of $\mu$ versus $n$ on the other hand, are difficult to reconcile. We would like to point out that a similar situation is found for InAs quantum wells, where an even larger Dingle ratio and a sublinear relationship between $\mu$ and $n$ are observed together [30]. On the one hand, we would like to note that interfaces in InSb quantum wells (as well as in InAs quantum wells) are rather rough compared to high-quality GaAs quantum wells. It is unclear in what way interface roughness scattering plays a role in our present experiments and how it would influence the relationship between mobility and density. On the other hand, we speculate that spin-orbit interactions which are relevant in InSb could play a role here.

Repeating the measurement for $n=3.25 \times 10^{11} \mathrm{~cm}^{-2}$ and $n=2.9 \times 10^{11} \mathrm{~cm}^{-2}$, we find the effective mass to be constant and the ratio $\tau_{D} / \tau_{q}$ is always $\sim 29$. Combining this with the coincidence measurement, we conclude that $\left|g^{*}\right|=$ $35 \pm 4$. Furthermore, we obtain the same results for another set of coincidence measurements at $90 \mathrm{mK}$ with a lower carrier density $\left(n=2.88 \times 10^{11} \mathrm{~cm}^{-2}\right)$.

We compare our results with previous works. The change of the spin polarization $P$ is defined as $P=r / v$. In Ref. [12], the spin polarization $P$ varied over a wide range, from 0.07 to 1 . By extrapolating the measured $g$ factor to $P=0$, the bare $g$ factor was derived to have a value of $g^{*}=29$ for 30-nm QWs and $g^{*}=19.5-21.2$ for $15-n m$ QWs. A similar analysis was made in Ref. [13], where $P$ ranged from 0.07 to 1 for $v=7-14$ and a slightly higher effective $g$ factor was derived. Due to the high mobility of our sample, our coincidence measurement is performed in a regime of large filling factors where the exchange enhancement can be neglected. In our measurement, the Landau levels in the InSb 2DEG are distinguishable from $r=1 / 2$ to 2 , and the tilt angle dependence of the $\mathrm{SdH}$ oscillations has been investigated from $v=5$ to 28 . These give a range of $P$ from 0.018 to 0.2 , where there is no obvious $\left|g^{*}\right|-B_{\perp}$ dependence observed. Thus, we conclude from our estimation that the extracted value of $\left|g^{*}\right|$ is basically the bare effective $g$ factor of the 2DEGs in InSb QWs.

Our measurement results can be quantitatively compared to multiband $\boldsymbol{k} \cdot \boldsymbol{p}$ theory where both the $s$ - and $p$-like bands are considered [31]. Due to the confinement of the QW, the band gap of InSb will have an increase of $E_{g}=\pi^{2} \hbar^{2} / 2 L^{2} m_{\text {bulk }}^{*}=$ $61 \mathrm{meV}$ based on the infinite potential well approximation, where $m_{\text {bulk }}^{*} \approx 0.014 m_{e}$ is the electron effective mass of bulk $\operatorname{InSb}[26,31]$ and $L=21 \mathrm{~nm}$ is the width of the QW. Thus, we can calculate, as in Refs. [26,31],

$$
\begin{aligned}
\frac{m_{e}}{m_{0}^{*}}= & 1+\frac{1}{3} \frac{2 m_{e} P^{2}}{\hbar^{2}}\left(\frac{2}{E_{0}+E_{g}}+\frac{1}{E_{0}+\Delta_{0}+E_{g}}\right) \\
& -\frac{1}{3} \frac{2 m_{e} P^{\prime 2}}{\hbar^{2}}\left(\frac{2}{E_{0}^{\prime}-E_{0}+\Delta_{0}^{\prime}}+\frac{1}{E_{0}^{\prime}-E_{0}}\right)
\end{aligned}
$$

and

$$
\begin{aligned}
g_{0}^{*}= & -\frac{2}{3} \frac{2 m_{e} P^{2}}{\hbar^{2}}\left(\frac{1}{E_{0}+E_{g}}-\frac{1}{E_{0}+E_{g}+\Delta_{0}}\right) \\
& +\frac{2}{3} \frac{2 m_{e} P^{\prime 2}}{\hbar^{2}}\left(\frac{1}{E_{0}^{\prime}-E_{0}}-\frac{1}{E_{0}^{\prime}-E_{0}+\Delta_{0}^{\prime}}\right),
\end{aligned}
$$




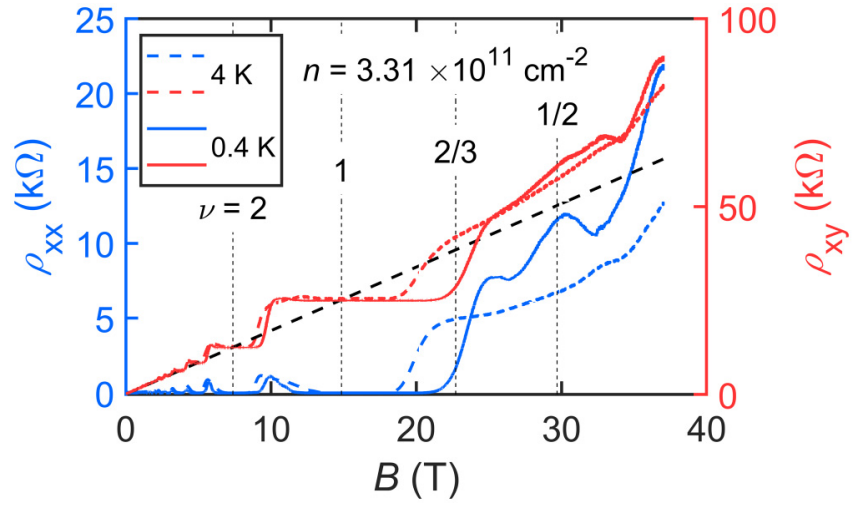

FIG. 5. The $\rho_{x x}$ and $\rho_{x y}$ measurement of the InSb QW grown on a GaSb wafer in a large magnetic field range at the temperatures of 4 and $0.4 \mathrm{~K}$. The dashed line is an extrapolation of the Hall trace in the small magnetic field.

where $m_{0}^{*}$ and $g_{0}^{*}$ are the theoretical electron effective mass and effective $g$ factor in the QW [26]. Here, we adopt the band-edge parameters $E_{0}=0.24 \mathrm{eV}, E_{0}^{\prime}=3.16 \mathrm{eV}$, $\Delta_{0}=0.82 \mathrm{eV}, \Delta_{0}^{\prime}=0.33 \mathrm{eV}, P=0.964 \mathrm{eV} \mathrm{nm}$, and $P^{\prime}=$ $0.632 i \mathrm{eVnm}$ from Refs. [26,31]. The interpretation of these parameters can also be found in Ref. [32]. The calculation shows that the theoretical effective mass of the electrons in the QW has a value of $m_{0}^{*}=0.0152 m_{e}$ and the effective $g$-factor has a value of $g_{0}^{*}=-38$, which agrees with our measurement results. We also find that the $s$-like bands and confinement of QWs are the dominant factors in our sample that determine effective mass and $g$ factor.

The high mobility of our sample motivates us to probe the behavior of the 2DEGs in an even higher magnetic field, where $v<1$. We perform a magnetotransport measurement on another Hall bar sample with the same quality, where the heterostructure is grown on a GaSb wafer with the same growth and microfabrication. The mobility is $300000 \mathrm{~cm}^{2} /(\mathrm{V} \mathrm{s})$ and the carrier density is $3.31 \times$ $10^{11} \mathrm{~cm}^{-2}$. As shown in Fig. 5, measurements at 4 and $0.4 \mathrm{~K}$ reveal similar $\rho_{x x}$ and $\rho_{x y}$ behaviors at $\nu<1$, where the Ohmic contacts still work properly. This implies that the 2DEG is not in an insulating phase. Despite the high quality of the sample, no fractional quantum Hall effect features are observable. The broad local minima of $\rho_{x x}$ at $B=27$ and $33 \mathrm{~T}$ are far from the estimated magnetic field values corresponding to the filling factor values $v=2 / 3$ and $1 / 2$ and their locations strongly depend on temperature. Besides, $\rho_{x y}$ at $v<1$ increases above the classical limit, which is indicated by the dashed line in Fig. 5. Even though the InSb QW sample measured here exhibits comparably high mobility as shown in Ref. [22], the impact of disorder or other effects on the transport behavior in the extreme quantum limit needs to be further investigated. This raises the question of whether material-specific properties are responsible for the observations since a similar behavior has been observed before in an InSb QW with a mobility of $130000 \mathrm{~cm}^{2} /(\mathrm{V} \mathrm{s})$ [33].

For experiments at $B=0$ the so-called $r_{s}$ parameter, which is the ratio between electron-electron interaction energy and kinetic energy, is a measure for the occurrence of effects that are related to electron-electron interactions. The $r_{s}$ parameter for InSb is rather small compared to other materials (GaAs, graphene, and even InAs) because of the small effective mass in InSb. The situation in the fractional quantum Hall regime is more complex since the relevant distance for electron-electron interactions is the magnetic length $l_{B}=(\hbar / e B)^{1 / 2}$ which is independent of material parameters. Still, the ratio between the Coulmb energy $e^{2} /\left(4 \pi \varepsilon_{0} \varepsilon l_{B}\right)$ and the kinetic energy $\frac{1}{2} \hbar \omega_{c}$ in the quantum limit is comparably small in our InSb sample $(0.11$ at $20 \mathrm{~T})$ as compared to, e.g., GaAs (0.59 at $20 \mathrm{~T})$. This cannot, however, fully explain the fact that we do not observe features of the fractional quantum Hall effect in high-mobility InSb quantum wells. We speculate that it is the complex interplay of interaction strength (small effective mass, high magnetic field) and disorder (see discussion on Dingle ratio, mobility versus density dependence, interface quality) that results in the absence of features related to the fractional quantum Hall effect in our experiments.

In conclusion, we present a measurement of the bare $g$ factor of an InSb QW with tunable density. Due to the high mobility, our measurement is accomplished in a low magnetic field regime where many-body physics can be neglected. Together with a precise measurement of the effective mass, the bare effective $g$ factor of our InSb QW is determined to be $\left|g^{*}\right|=35 \pm 4$. Furthermore, probing of the electron transport behavior for filling factors $v<1$ is presented, where the fractional quantum Hall effect is not observed.

We thank Dr. F. K. de Vries and Dr. P. Rickhaus for fruitful discussions. We acknowledge the support of HFMLRU/NWO-I, member of the European Magnetic Field Laboratory (EMFL). This work was supported by the Swiss National Science Foundation through the National Center of Competence in Research (NCCR) Quantum Science and Technology.
[1] R. L. Kallaher, J. J. Heremans, N. Goel, S. J. Chung, and M. B. Santos, Spin-orbit interaction determined by antilocalization in an InSb quantum well, Phys. Rev. B 81, 075303 (2010).

[2] G. A. Khodaparast, R. E. Doezema, S. J. Chung, K. J. Goldammer, and M. B. Santos, Spectroscopy of Rashba spin splitting in InSb quantum wells, Phys. Rev. B 70, 155322 (2004).

[3] M. Leontiadou, K. Litvinenko, A. Gilbertson, C. Pidgeon, W. Branford, L. Cohen, M. Fearn, T. Ashley, M. Emeny, B. Murdin et al., Experimental determination of the Rashba coefficient in InSb/InAlSb quantum wells at zero magnetic field and elevated temperatures, J. Phys.: Condens. Matter 23, 035801 (2011).

[4] A. M. Gilbertson, W. R. Branford, M. Fearn, L. Buckle, P. D. Buckle, T. Ashley, and L. F. Cohen, Zero-field spin splitting and spin-dependent broadening in high-mobility InSb/In1-xAlxSb asymmetric quantum well heterostructures, Phys. Rev. B 79, 235333 (2009).

[5] J. Orr, P. Buckle, M. Fearn, C. Storey, L. Buckle, and T. Ashley, A surface-gated InSb quantum well single electron transistor, New J. Phys. 9, 261 (2007). 
[6] O. Madelung, U. Roessler, and M. Schulz, Landolt-BernsteinGroup III Condensed Matter (Springer, Berlin, 2000), Vol. 41D.

[7] T. Ashley, A. Dean, C. Elliott, G. Pryce, A. Johnson, and H. Willis, Uncooled high-speed InSb field-effect transistors, Appl. Phys. Lett. 66, 481 (1995).

[8] H. Chen, J. Heremans, J. Peters, A. Govorov, N. Goel, S. Chung, and M. Santos, Spin-polarized reflection in a twodimensional electron system, Appl. Phys. Lett. 86, 032113 (2005).

[9] I. Zutic, J. Fabian, and S. D. Sarma, Spintronics: Fundamentals and applications, Rev. Mod. Phys. 76, 323 (2004).

[10] C. T. Ke, C. M. Moehle, F. K. de Vries, C. Thomas, S. Metti, C. R. Guinn, R. Kallaher, M. Lodari, G. Scappucci, T. Wang et al., Ballistic superconductivity and tunable $\pi$-junctions in InSb quantum wells, Nat. Commun. 10, 3764 (2019).

[11] Y. Oreg, G. Refael, and F. von Oppen, Helical Liquids and Majorana Bound States in Quantum Wires, Phys. Rev. Lett. 105, 177002 (2010).

[12] B. Nedniyom, R. J. Nicholas, M. T. Emeny, L. Buckle, A. M. Gilbertson, P. D. Buckle, and T. Ashley, Giant enhanced g-factors in an InSb two-dimensional gas, Phys. Rev. B 80, 125328 (2009).

[13] K. F. Yang, H. W. Liu, T. D. Mishima, M. B. Santos, K. Nagase, and Y. Hirayama, Nonlinear magnetic field dependence of spin polarization in high-density two-dimensional electron systems, New J. Phys. 13, 083010 (2011).

[14] H. A. Nilsson, P. Caroff, C. Thelander, M. Larsson, J. B. Wagner, L.-E. Wernersson, L. Samuelson, and H. Xu, Giant, level-dependent g-factors in InSb nanowire quantum dots, Nano Lett. 9, 3151 (2009).

[15] H. A. Nilsson, O. Karlström, M. Larsson, P. Caroff, J. N. Pedersen, L. Samuelson, A. Wacker, L.-E. Wernersson, and H. Q. Xu, Correlation-Induced Conductance Suppression at Level Degeneracy in a Quantum Dot, Phys. Rev. Lett. 104, 186804 (2010).

[16] F. Qu, J. van Veen, F. K. de Vries, A. J. Beukman, M. Wimmer, W. Yi, A. A. Kiselev, B.-M. Nguyen, M. Sokolich, M. J. Manfra et al., Quantized conductance and large $g$-factor anisotropy in InSb quantum point contacts, Nano Lett. 16, 7509 (2016).

[17] T. Masuda, K. Sekine, K. Nagase, K. S. Wickramasinghe, T. D. Mishima, M. B. Santos, and Y. Hirayama, Transport characteristics of InSb trench-type in-plane gate quantum point contact, Appl. Phys. Lett. 112, 192103 (2018).

[18] G. W. Winkler, D. Varjas, R. Skolasinski, A. A. Soluyanov, M. Troyer, and M. Wimmer, Orbital Contributions to the Electron g-Factor in Semiconductor Nanowires, Phys. Rev. Lett. 119, 037701 (2017).

[19] J. C. Chokomakoua, N. Goel, S. J. Chung, M. B. Santos, J. L. Hicks, M. B. Johnson, and S. Q. Murphy, Ising quantum
Hall ferromagnetism in InSb-based two-dimensional electronic systems, Phys. Rev. B 69, 235315 (2004).

[20] V. Mourik, K. Zuo, S. M. Frolov, S. R. Plissard, E. P. A. M. Bakkers, and L. P. Kouwenhoven, Signatures of majorana fermions in hybrid superconductor-semiconductor nanowire devices, Science 336, 1003 (2012).

[21] I. Kulesh, C. T. Ke, C. Thomas, S. Karwal, C. M. Moehle, S. Metti, R. Kallaher, G. C. Gardner, M. J. Manfra, and S. Goswami, Stable Quantum Dots in an InSb Two-Dimensional Electron Gas, Phys. Rev. Appl. 13, 041003 (2020).

[22] C. A. Lehner, T. Tschirky, T. Ihn, W. Dietsche, J. Keller, S. Fält, and W. Wegscheider, Limiting scattering processes in highmobility InSb quantum wells grown on GaSb buffer systems, Phys. Rev. Mater. 2, 054601 (2018).

[23] Z. Lei, C. A. Lehner, E. Cheah, M. Karalic, C. Mittag, L. Alt, J. Scharnetzky, W. Wegscheider, T. Ihn, and K. Ensslin, Quantum transport in high-quality shallow InSb quantum wells, Appl. Phys. Lett. 115, 012101 (2019).

[24] H. W. Schumacher, A. Nauena, U. Zeitler, R. J. Haug, P. Weitz, A. G. M. Jansen, and F. Schäfflerd, Anomalous coincidences between valley split Landau levels in a $\mathrm{Si} / \mathrm{SiGe}$ heterostructure, Phys. B: Condens. Matter 256-258, 260 (1998).

[25] S. Brosig, K. Ensslin, A. G. Jansen, C. Nguyen, B. Brar, M. Thomas, and H. Kroemer, InAs-AlSb quantum wells in tilted magnetic fields, Phys. Rev. B 61, 13045 (2000).

[26] T. Ihn, Semiconductor Nanostructures: Quantum States and Electronic Transport (Oxford University Press, Oxford, New York, 2010).

[27] P. T. Coleridge, Small-angle scattering in two-dimensional electron gases, Phys. Rev. B 44, 3793 (1991).

[28] S. Das Sarma and F. Stern, Single-particle relaxation time versus scattering time in an impure electron gas, Phys. Rev. B 32, 8442 (1985).

[29] V. Umansky, R. de-Picciotto, and M. Heiblum, Extremely highmobility two dimensional electron gas: Evaluation of scattering mechanisms, Appl. Phys. Lett. 71, 683 (1997).

[30] T. Tschirky, S. Mueller, Ch. A. Lehner, S. Falt, T. Ihn, K. Ensslin, and W. Wegscheider, Scattering mechanisms of highest-mobilityInAs $/ \mathrm{Al}_{x} \mathrm{Ga}_{1-x}$ Sbquantum wells, Phys. Rev. B 95, 115304 (2017).

[31] R. Winkler, Spin Orbit Coupling Effect in Two Dimensional Electron and Hole Systems (Springer, Berlin, Heidelberg, 2003).

[32] H. Mayer and U. Rössler, Spin splitting and anisotropy of cyclotron resonance in the conduction band of GaAs, Phys. Rev. B 44, 9048 (1991).

[33] K. J. Goldammer, S. J. Chung, W. K. Liu, M. B. Santos, J. L. Hicks, S. Raymond, and S. Q. Murphy, High-mobility electron systems in remotely-doped InSb quantum wells, J. Cryst. Growth 201-202, 753 (1999). 